www.nature.com/pj

\title{
Interaction between living cells and polymeric particles: potential application of ionic liquid for evaluating the cellular uptake of biodegradable polymeric particles composed of poly(amino acid)
}

\author{
Fumiaki Shima ${ }^{1}$, Takami Akagi ${ }^{1}$, Manami Shudo, Eiko Mochizuki, Tetsuya Tsuda, Susumu Kuwabata and \\ Mitsuru Akashi ${ }^{1}$
}

The cellular uptake of biodegradable particles as drug or vaccine carriers was observed by scanning electron microscopy employing an ionic liquid. The samples were observed by simply dipping them into the ionic liquid, and high-resolution images (sub-nanometer order) were achieved. The cellular uptake of polymeric nanoparticles (NPs) composed of biodegradable amphiphilic polymers was observed using an ionic liquid method for the first time. In particular, these NPs were observed when the quantum dots were immobilized on the NPs. In addition, when the cells were incubated with microparticles (MPs), the filopodia that covered the MPs were observed, and the cellular uptake of the MPs was evaluated in a time-dependent manner. This ionic liquid method is a promising technique for evaluating the cellular uptake behavior of drug or vaccine carriers. This method also provides a strategy for observing carriers that are sensitive to conventional pretreatment conditions by choosing a suitable ionic liquid.

Polymer Journal (2015) 47, 631-638; doi:10.1038/pj.2015.40; published online 1 July 2015

\section{INTRODUCTION}

Recently, nanotechnology has been applied for the development of safe and effective medicines and vaccines. In particular, drug delivery systems using polymeric nanoparticles (NPs) and/or microparticles (MPs) have been extensively studied as important carriers to deliver drugs or vaccine antigens, such as small molecules, peptides and proteins. ${ }^{1,2}$ The NPs and MPs with entrapped antigens represent an interesting approach for controlling the biodistribution and intracellular behavior of the antigens and optimizing the desired immune responses. ${ }^{3,4}$ To maximize the immune response, the NPs and MPs have to be internalized into antigen presenting cells (APCs), such as macrophages and dendritic cells. ${ }^{5}$ The cellular uptake of these particles is the first and most important step in the immune response. To gain insight into the mechanisms of the immune response, fluorescence microscopy has been mainly used. Fluorescence labeled compounds and NPs are incubated with APCs, and their uptake and intracellular behaviors are visualized. ${ }^{6}$ These methods are advantageous due to the noninvasive, simple observation method and real-time imaging. ${ }^{7}$ However, there are also limitations, such as only information from the fluorescent labeling is collected without sufficient resolution to observe cellular structures or cellular uptake of particles on the nanometer scale. Therefore, imaging techniques that can observe objects on the nanometer scale are required.
To achieve this level of resolution, scanning electron microscopy (SEM) has been employed. This method can achieve sub-nanometer resolution and be used to evaluate subcellular structures. ${ }^{8}$ However, these observations can only be conducted under high vacuum conditions, and liquid may not be introduced into the vacuum chamber. Therefore, the cells are not in their native liquid state. Therefore, various observation methods have been employed to observe the cells under hydrated conditions. In recent years, wet samples have been successfully observed by introducing a small chamber enclosed by a thin membrane, which is transparent to the electron beam. ${ }^{9}$ These wet SEM and environmental SEM techniques allow for investigating aqueous samples under fully physiological conditions. Although the cells can be observed without staining, the resolution is insufficient to evaluate the cellular uptake of the carriers, and sophisticated devices would be required. ${ }^{10,11}$ In addition, to observe the cellular uptake of NPs in detail without the charge-up effect, the samples must be subjected to pretreatments, such as coating or staining with metals or carbon. These steps inevitably change the shape and delicate structures of the cells, and extended pretreatment and imaging times are required. Because conventional procedures do not quickly produce high-resolution images a technique that uses simple pretreatment methods with the ability to record highresolution images is needed. 


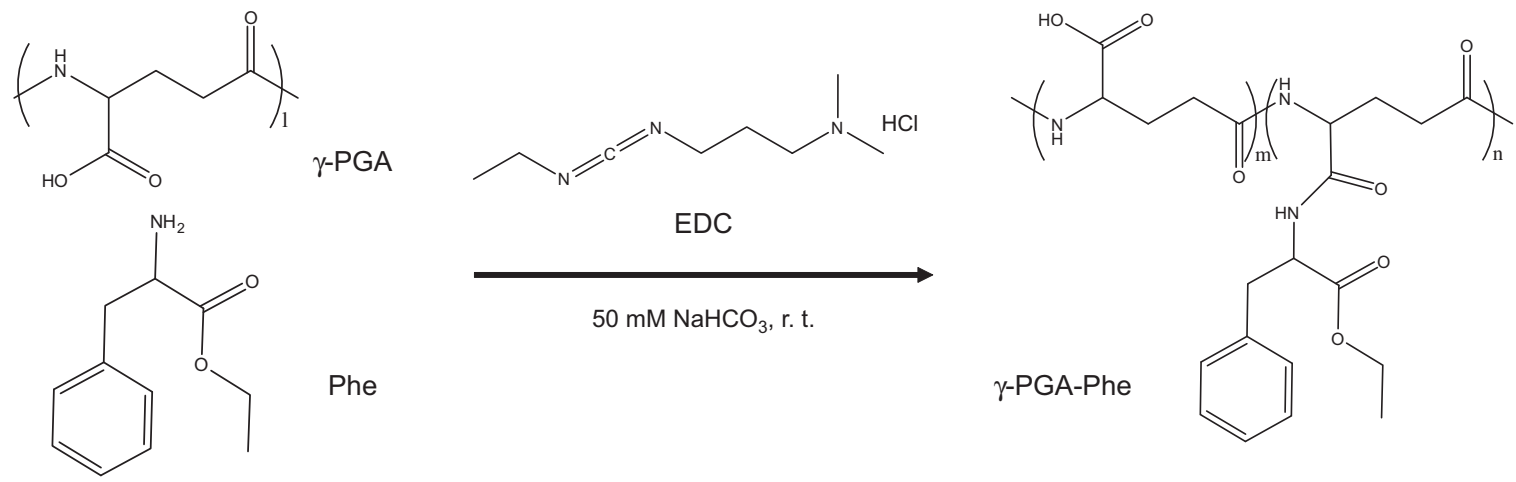

Scheme 1 Synthetic scheme for the $\gamma$-PGA-Phe copolymer.

Ionic liquids composed of cationic and anionic ions exhibit several attractive features, such as having a liquid phase at ambient temperature, $^{12}$ negligible vapor pressure $^{13}$ and high ionic conductivity. ${ }^{14}$ We demonstrated that ionic liquids can be observed by SEM without the charge-up effect even in the absence of a metal or carbon coating, which indicated that the ionic liquid behaves as an electronically conducting material. ${ }^{15} \mathrm{We}$ observed samples in the wet state by replacing the water with an ionic liquid. ${ }^{16,17}$ In addition, the cellular uptake of particles was successfully observed by SEM using an ionic liquid, which demonstrated that the ionic method exhibited the potential for use as a novel approach for evaluating the uptake behavior of vaccine carriers by APCs. ${ }^{18}$

In a previous study, we prepared NPs composed of hydrophobically modified poly $(\gamma$-glutamic acid) ( $\gamma$-PGA) to develop effective vaccines. ${ }^{19}$ The encapsulated antigens in these NPs were efficiently taken up by APCs, and an antigen-specific immune response was induced. ${ }^{20}$ The size of the NPs was controlled by the preparation method to prepare unimolecular (unimer) NPs to $\sim 200-\mathrm{nm}$ sized NPs. $^{21,22}$ These results strongly suggested that hydrophobically modified $\gamma$-PGA NPs has the potential to be a candidate for use in an antigen delivery system.

In this study, the ionic liquid method was extended for use in SEM observations of the cellular uptake of biodegradable vaccine carriers, such as NPs and MPs, by APCs. We investigated the cellular uptake of vaccine carriers by SEM using an ionic liquid and observed the uptake processes of NPs and MPs under wet conditions.

\section{EXPERIMENTAL PROCEDURE}

\section{Materials}

$\gamma$-PGA $\left(M_{\mathrm{w}}=500000\right)$, chloroform, 1-ethyl-3-(3-dimethylaminopropyl) carbodiimide (EDC), Dulbecco's modified Eagle's medium, dimethylsulfoxide, sodium chloride, phosphate-buffered saline (PBS) and ethanol were purchased from Wako Pure Chemical Industries (Osaka, Japan). L-phenylalanine ethyl ester (Phe), fetal bovine serum and fibronectin were purchased from SigmaAldrich (St Louis, MO, USA). The antibiotics ( 100 units $\mathrm{ml}^{-1}$ penicillin, 100 $\mu \mathrm{g} \mathrm{ml}^{-1}$ streptomycin and $0.25 \mu \mathrm{g} \mathrm{ml}^{-1}$ amphotericin B) were purchased from Nacalai Tesque (Kyoto, Japan). Mouse leukemic monocytes (that is, RAW264 cells) were purchased from Riken BRC Cell Bank (Ibaraki, Japan). The fluorine-doped tin oxide (FTO) glass was purchased from AGC Fabritech (Tokyo, Japan). 1-Ethyl-3-methylimidazolium tetrafluoroborate $\left([\mathrm{EMI}] \mathrm{BF}_{4}\right)$ and choline lactate were purchased from the Tokyo Chemical Industry (Tokyo, Japan). Qdot ITK: amino (PEG) quantum dots 525 were purchased from Thermo Fischer Scientific (Waltham, MA, USA). The silica particles $(1 \mu \mathrm{m})$ were purchased from COREFRONT (Tokyo, Japan).

\section{Synthesis of $\boldsymbol{\gamma}$-PGA-Phe copolymer}

$\gamma$-PGA ( 5 unit mmol) was hydrophobically modified by Phe $(5 \mathrm{mmol})$ using EDC ( 5 or $10 \mathrm{mmol}$ ) in a $50 \mathrm{~mm}$ sodium hydrogen carbonate solution $(100 \mathrm{ml})$ for $1 \mathrm{~h}$ on ice and then for $24 \mathrm{~h}$ at room temperature (Scheme 1). ${ }^{23}$ Then, the synthesized $\gamma$-PGA-Phe copolymer was dialyzed against water for 5 days and freeze dried for 3 days. The $\gamma$-PGA-Phe copolymer was confirmed by FT-IR spectroscopy (Spectrum 100, Perkin Elmer, Waltham, MA, USA) and ${ }^{1} \mathrm{H}$ NMR (ECS-400, JEOL, Tokyo, Japan).

\section{Preparation of $\boldsymbol{\gamma}$-PGA-Phe NPs}

$\gamma$-PGA-Phe NPs were prepared according to previously reported protocol. ${ }^{24}$ Briefly, $\gamma$-PGA-Phe $\left(10 \mathrm{mg} \mathrm{ml}^{-1}\right.$ in dimethylsulfoxide) was added to the same volume of sodium chloride solution. The $200 \mathrm{~nm}$ sized NPs were prepared by adjusting the salt concentration to $0.2 \mathrm{M}$. Then, the resulting solutions were dialyzed for 3 days and lyophilized for 2 days. The $\gamma$-PGA-Phe NPs were dispersed into PBS, and their size distribution was measured using a dynamic light scattering method using a Zetasizer Nano ZS (Malvern Instruments, Malvern, UK). The morphology of the $\gamma$-PGA-Phe NPs was observed using SEM (JSM-6701 F, JEOL).

To achieve a high contrast image of the $\gamma$-PGA-Phe NPs, a quantum dot (QD) was immobilized on the $\gamma$-PGA-Phe NPs. The amine groupfunctionalized QDs were grafted to the carboxyl groups of $\gamma$-PGA-Phe NPs. Briefly, $5 \mathrm{mg} \mathrm{ml}^{-1}$ of the $\gamma$-PGA-Phe NPs in $20 \mathrm{~mm}$ phosphate buffer $(500 \mu \mathrm{l}$, $\mathrm{pH}$ 5.8) were incubated with $2 \mathrm{mg} \mathrm{ml}^{-1}$ EDC in $20 \mathrm{~mm}$ phosphate buffer $(500 \mu \mathrm{l}, \mathrm{pH} 5.8)$ at room temperature to activate the carboxyl groups. After centrifugation to remove the unreacted EDC, the $\gamma$-PGA-Phe NPs were dispersed in ultrapure water $(980 \mu \mathrm{l})$ and incubated with $8.5 \mu \mathrm{M}$ QDs $(20 \mu \mathrm{l})$ for $24 \mathrm{~h}$ at room temperature. After centrifugation, the QD-immobilized $\gamma$-PGA-Phe NPs (QD-NPs) were dispersed into PBS. The amount of immobilized QDs was determined by the fluorescence intensity of the QDs.

\section{Preparation of $\boldsymbol{\gamma}$-PGA-Phe MPs}

The $\gamma$-PGA-Phe MPs were prepared using the emulsion method. $30 \mu \mathrm{l}$ of a $\gamma$-PGA-Phe $\left(10 \mathrm{mg} \mathrm{ml}^{-1}\right.$ in chloroform) solution was added to $3 \mathrm{ml}$ of ultrapure water and pipetted several times to prepare the emulsion. After the chloroform was evaporated, the $\gamma$-PGA-Phe MPs were obtained. The morphology of the $\gamma$-PGA-Phe MPs was observed by SEM.

\section{Cell culture}

Mouse leukemic monocytes (that is, RAW264 cells) were used in the current study. The RAW264 cells were cultured in Dulbecco's modified Eagle's medium

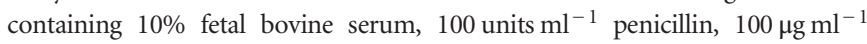
streptomycin and $0.25 \mu \mathrm{g} \mathrm{ml}^{-1}$ amphotericin B. The cells were maintained in $5 \% \mathrm{CO}_{2}$ at $37^{\circ} \mathrm{C}$.

\section{Uptake of NPs and MPs by RAW264 cells}

The FTO glass was dipped into a $40 \mu \mathrm{g} \mathrm{ml}^{-1}$ fibronectin solution for $1 \mathrm{~h}$. After washing with a $50 \mathrm{~mm}$ Tris- $\mathrm{HCl}$ buffer solution $(\mathrm{pH}=7.4)$, the fibronectin-coated FTO glass was placed into the 24 wells, and the RAW264 


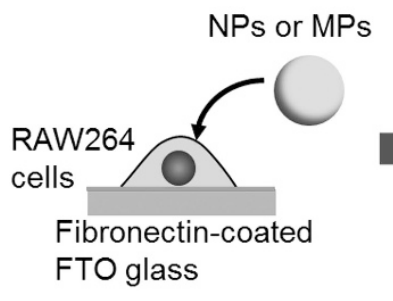

Fibronectin-coated

FTO glass

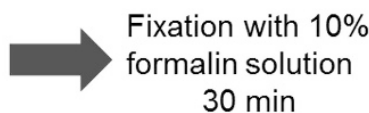

$30 \mathrm{~min}$

Scheme 2 Ionic liquid treatment procedure for SEM observation of the cellular uptake of NPs and MPs. A full color version of this figure is available at Polymer Journal online.

Table 1 Synthesis of the $\gamma$-PGA-Phe copolymers

\begin{tabular}{lccccc}
\hline 1 Run & $\gamma$-PGA (unit mmol) & Phe (mmol) & EDC (mmol) & Yield (\%) & G.D. (\%) \\
\hline 1 & 5 & 5 & 5 & 57 & 55 \\
2 & 5 & 5 & 10 & 65 & 65 \\
\hline
\end{tabular}

Abbreviations: EDC, 1-ethyl-3-(3-dimethylaminopropyl) carbodiimide; G.D., grafting degree; Phe, L-phenylalanine ethyl ester; $\gamma$-PGA, poly $(\gamma$-glutamic acid).

cells $\left(1 \times 10^{5}\right.$ cells per $0.5 \mathrm{ml}$ per well) were seeded and incubated for $24 \mathrm{~h}$. Then, the cells were incubated with $100 \mu \mathrm{g} \mathrm{ml}^{-1} \gamma$-PGA-Phe NPs, $\gamma$-PGA-Phe MPs or silica particles for the determined time period at $37^{\circ} \mathrm{C}$. After incubation, the cells were fixed with a $10 \%$ formalin solution for $30 \mathrm{~min}$ at room temperature and finally washed three times with PBS.

\section{Stability of $\boldsymbol{\gamma}$-PGA-Phe NPs in ionic liquid}

The $\gamma$-PGA-Phe NPs $\left(0.1 \mathrm{mg} \mathrm{ml}^{-1}\right.$ in PBS) were mixed with $0.5 \mathrm{wt} \%$ $[\mathrm{EMI}] \mathrm{BF}_{4}$ or $2 \mathrm{wt} \%$ choline lactate solution and casted to the cover glass. After vacuuming to remove the water, the sample was observed by SEM, and the morphology of the $\gamma$-PGA-Phe NPs was evaluated.

The effect of the ionic liquid treatment on the observation of cellular uptake of the $\gamma$-PGA-Phe NPs was evaluated using fluorescent-labeled $\gamma$-PGA-Phe NPs. The Alexa488-labeled $\gamma$-PGA-Phe NPs (Alexa488-NPs) were prepared according to a previously published protocol. ${ }^{25}$ The RAW264 cells were incubated with $0.1 \mathrm{mg} \mathrm{ml}^{-1}$ Alexa488-NPs for $1 \mathrm{~h}$ at $37^{\circ} \mathrm{C}$. After being thoroughly washed with PBS and fixed with a 10\% formalin solution, the sample was dipped into a $1 \mathrm{wt} \%$ choline lactate solution for $15 \mathrm{~s}$. The internalized Alexa488-NPs were observed using confocal fluorescence microscopy (DSU-IX81-SET fluorescence microscope, Olympus, Tokyo, Japan).

\section{Ionic liquid treatment for SEM observation}

In this study, $[\mathrm{EMI}] \mathrm{BF}_{4}$ and choline lactate were employed. [EMI]BF 4 or choline lactate was mixed with pure water at the desired concentration before use and vortexed for several seconds. Then, the sample was dipped into the ionic liquid solution for $15 \mathrm{~s}$, and any excess ionic liquid was absorbed by careful blotting with paper. The back side of the FTO glass was evenly pasted onto electrically conductive tape (Nisshin EM, Tokyo, Japan) that was placed on an aluminum sample stub for SEM (Scheme 2). Conductive staining, dehydration, drying and coating were not performed in this procedure. For the evaluation of the effect of ionic liquid concentration on the observation, the sample was dipped into a $0.5,2$ or $5 \mathrm{wt} \%$ ionic liquid solution for $15 \mathrm{~s}$ before observation by SEM.

For comparison to the conventional SEM method, the samples were dehydrated using 50,60, 70, 80, 90 and 100\% graded ethanol. The samples were transferred to $t$-butyl alcohol. Then, the samples were frozen at $-15^{\circ} \mathrm{C}$ and freeze dried for $1 \mathrm{~h}$. After being coated with osmium, the samples were observed by SEM.

\section{RESULTS AND DISCUSSION}

Preparation of $\gamma$-PGA-Phe NPs and MPs

The $\gamma$-PGA-Phe copolymers were synthesized using a coupling reaction between the amine group of Phe and the carboxylic groups of $\gamma$-PGA employing EDC. The grafting degree of Phe was determined by ${ }^{1} \mathrm{H}$ NMR spectroscopy using the integrals of the methylene peaks of $\gamma$-PGA and the phenyl peaks of Phe. The grafting degree was controlled between 55 and $65 \%$ by changing the molar ratio of EDC to $\gamma$-PGA from 1 to 2 (Table 1). In this study, we used a $\gamma$-PGA-Phe copolymer with a $55 \%$ grafting degree for preparing the NPs and a $65 \%$ grafting degree for preparing the MPs.

The $\gamma$-PGA-Phe NPs were prepared according to a previously reported protocol. ${ }^{24}$ The $\gamma$-PGA-Phe copolymers were able to form NPs due to their amphiphilic properties. The main driving force was the inter/intramolecular hydrophobic interactions between the hydrophobic side chains and hydrogen bonding. In this study, 200-nm sized $\gamma$-PGA-Phe NPs were prepared for the following experiment by adjusting the salt concentration to $0.2 \mathrm{M}$. On the basis of the dynamic light scattering measurement and SEM observation, the $\gamma$-PGA-Phe NPs were monodispersed with a spherical morphology (Figure 1). To achieve a high contrast image of $\gamma$-PGA-Phe NPs, a QD was immobilized on the $\gamma$-PGA-Phe NPs (QD-NPs). The amine group-functionalized QD was immobilized on the $\gamma$-PGA-Phe NPs using a coupling reaction between the amine group of the QD and the carboxyl groups in the $\gamma$-PGA-Phe NPs. On the basis of the fluorescence intensity, $\sim 11$ QDs were immobilized on a single $\gamma$-PGA-Phe NP (data not shown). The size of the QD-NPs was comparable to the $\gamma$-PGA-Phe NPs without QDs. These QD-NPs were used in the following experiments.

To prepare the $\gamma$-PGA-Phe MPs, we employed an emulsion method. $\gamma$-PGA-Phe was dissolved in chloroform $\left(10 \mathrm{mg} \mathrm{ml}^{-1}\right)$, added to the ultrapure water and pipetted several times to form an emulsion (Figure 2a). After evaporation of the chloroform, $\gamma$-PGA-Phe formed spherical microparticles. The volume ratio of the $\gamma$-PGA-Phe solution to ultrapure water was critical for the formation of microparticles. By adjusting the $\gamma$-PGA-Phe solution to ultrapure water ratio to $1: 100, \gamma$-PGA-Phe formed monodispersed microparticles (Figure 2b). On the basis of the SEM images, the size of the MPs was determined to be $4.6 \pm 1.2 \mu \mathrm{m}(n=20)$.

\section{Optimization of the ionic liquid concentration for SEM observation} For a detailed evaluation of the cellular uptake of vaccine carriers, the ionic liquid concentration is crucial. Therefore, the effect of the ionic liquid concentration on the observation was evaluated by varying the concentration of the ionic liquid solution. The cells, which took up

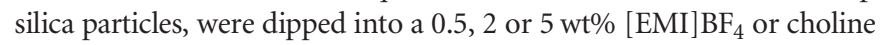
lactate solution for $15 \mathrm{~s}$ before observation by SEM (Figure 3 ). In the ionic liquid pretreatment, the ionic liquid was dissolved in water. Because $[\mathrm{EMI}] \mathrm{BF}_{4}$ and choline lactate are viscous, it is quite difficult to thinly coat the sample when the sample is dipped directly into undiluted ionic liquids. However, when ionic liquids are dissolved in water, the viscosity decreases, and the sample can be coated with a thin ionic liquid layer. When the sample was dipped into a $5 \mathrm{wt} \%$ ionic 


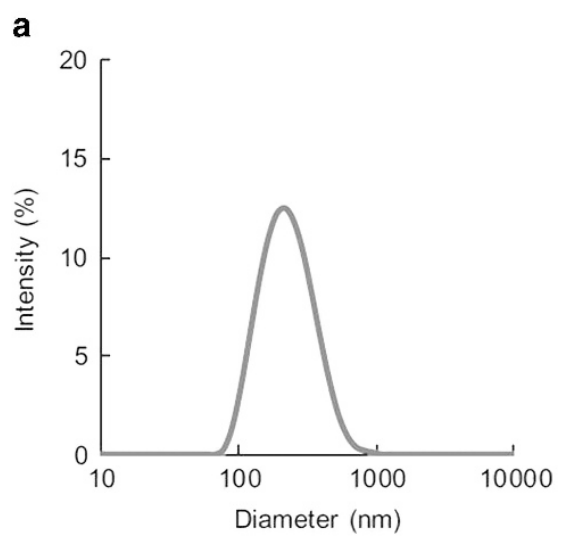

b

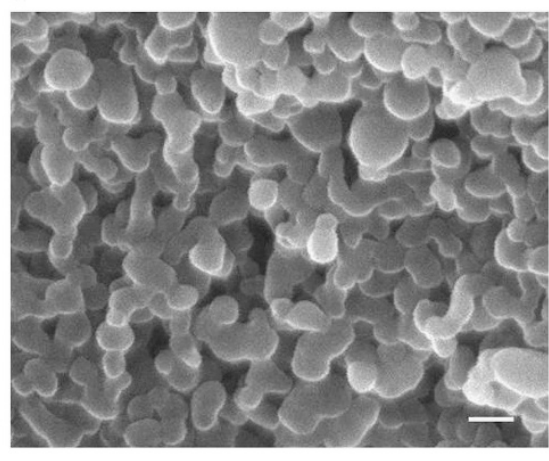

Figure 1 (a) Size distribution of the $\gamma$-PGA-Phe NPs measured by dynamic light scattering in PBS. (b) SEM observation of the $\gamma$-PGA-Phe NPs. The scale bar represents $100 \mathrm{~nm}$. A full color version of this figure is available at Polymer Journal online.

b

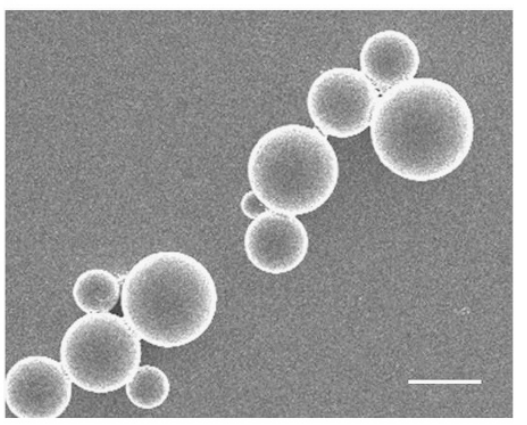

Figure 2 (a) Preparation of the $\gamma$-PGA-Phe MPs. (b) SEM image of the $\gamma$-PGA-Phe MPs. The scale bar represents $5 \mu \mathrm{m}$. A full color version of this figure is

a

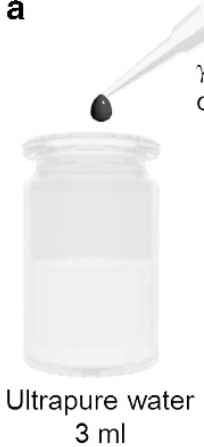
available at Polymer Journal online.

\section{$\gamma$-PGA-Phe in chloroform}

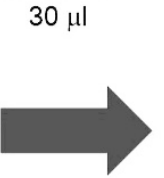

Pipetting
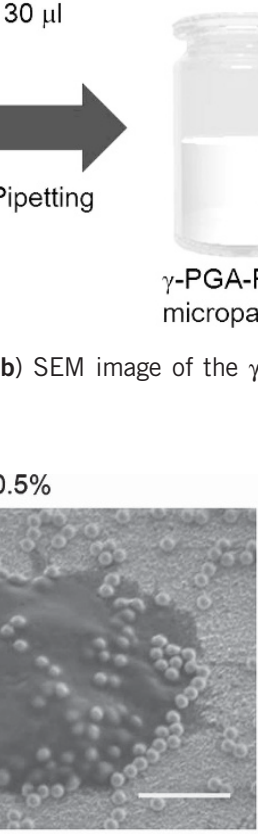

$\mathrm{EMI}^{-\mathrm{BF}_{4}}$<smiles></smiles>

Choline lactate

$\overbrace{\mathrm{OH}}$
$0.5 \%$
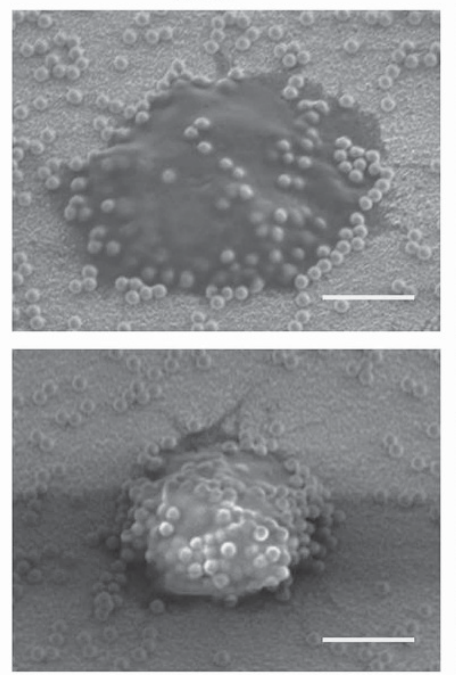

$2 \%$
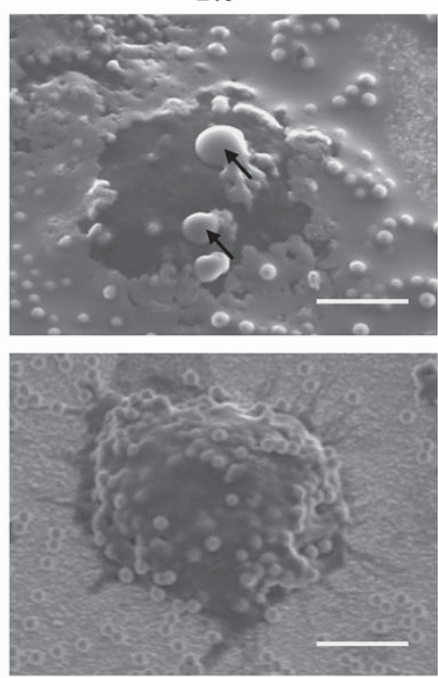

$5 \%$
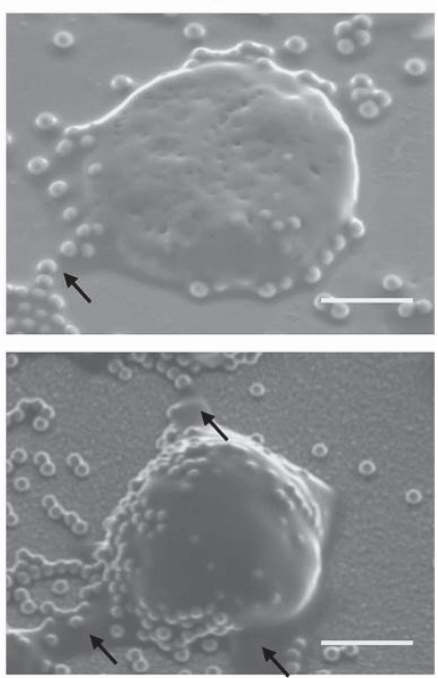

Figure 3 Effects of the ionic liquid concentration on the observation of the cells. The cells were dipped into a $0.5,2$ or 5 wt $\%$ ionic liquid solution for $15 \mathrm{~s}$ before SEM observation. The scale bars represent $5 \mu \mathrm{m}$. The arrows show the droplets of the ionic liquid.

liquid, the droplets of ionic liquid appeared around the cells, which indicated that the amount of ionic liquid was too large to observe the cellular uptake in detail. This phenomenon was also observed when the concentration of $\left[\mathrm{EMI}^{\mathrm{B}} \mathrm{BF}_{4}\right.$ was $2 \mathrm{wt} \%$ (Figure 3, arrows). When the concentration of choline lactate was $0.5 \mathrm{wt} \%$, the sample exhibited the charge-up effect. Therefore, we determined that the optimal concentration of $[\mathrm{EMI}] \mathrm{BF}_{4}$ and choline lactate were 0.5 and $2 \mathrm{wt} \%$, respectively. 
To determine the potential of a ionic liquid method, the cellular uptake was observed by phase contrast microscopy (control) or SEM using either the ionic liquid method or the conventional method (Figure 4). When the sample was observed by the conventional method, the size of the cells decreased (Figures $4 a, b$ and e), which was due to the shrinkage of the cells during the dehydration pretreatment hindering the detailed observation of the cellular uptake. In contrast, the size of the cells was comparable to the control when the ionic liquid method was employed (Figures $4 \mathrm{c}-\mathrm{e}$ ). The morphology of the cells observed in $[\mathrm{EMI}] \mathrm{BF}_{4}$ and choline lactate was the same as the control, and no damage, such as shrinkage or blebbing of the cells, was observed. Therefore, the ionic liquid method is a promising strategy for observing the cellular uptake of vaccine carriers without serious damage.

\section{Stability of the $\gamma$-PGA-Phe NPs in the ionic liquid}

To observe the cellular uptake of the $\gamma$-PGA-Phe NPs by SEM using the ionic liquid method, the $\gamma$-PGA-Phe NPs must be stable in the ionic liquid. In this study, the $\gamma$-PGA-Phe NPs were mixed with a
$0.5 \mathrm{wt} \%[\mathrm{EMI}] \mathrm{BF}_{4}$ or $2 \mathrm{wt} \%$ choline lactate solution, and the stability was evaluated by SEM. Because the ionic liquids exhibit electron conductivity, the sample was observed without a metal or carbon coating. In Figure 5a, the $\gamma$-PGA-Phe NPs exhibited a spherical morphology when mixed with choline lactate, which demonstrated that the $\gamma$-PGA-Phe NPs are stable in the ionic liquid and could be observed using the ionic liquid method. However, the $\gamma$-PGA-Phe

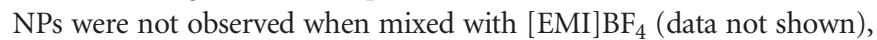
which indicated the collapse of the $\gamma$-PGA-Phe NPs. These results may be due to the interactions between the ionic liquids and the $\gamma$-PGAPhe copolymers. The $\gamma$-PGA-Phe NPs are formed by inter/intramolecular hydrophobic interactions between hydrophobic side chains and hydrogen bonding. The aromatic ring in [EMI] might interact with the phenyl group in the hydrophobic side chains and interrupt the hydrophobic interactions between the side chains. These interactions would hinder the driving forces of self-assembly of the $\gamma$-PGA-Phe copolymers, resulting in the collapse of the $\gamma$-PGA-Phe NPs. In addition, the $\gamma$-PGA-Phe NPs were slightly larger than those observed by the conventional method, which suggested that the NPs might exist
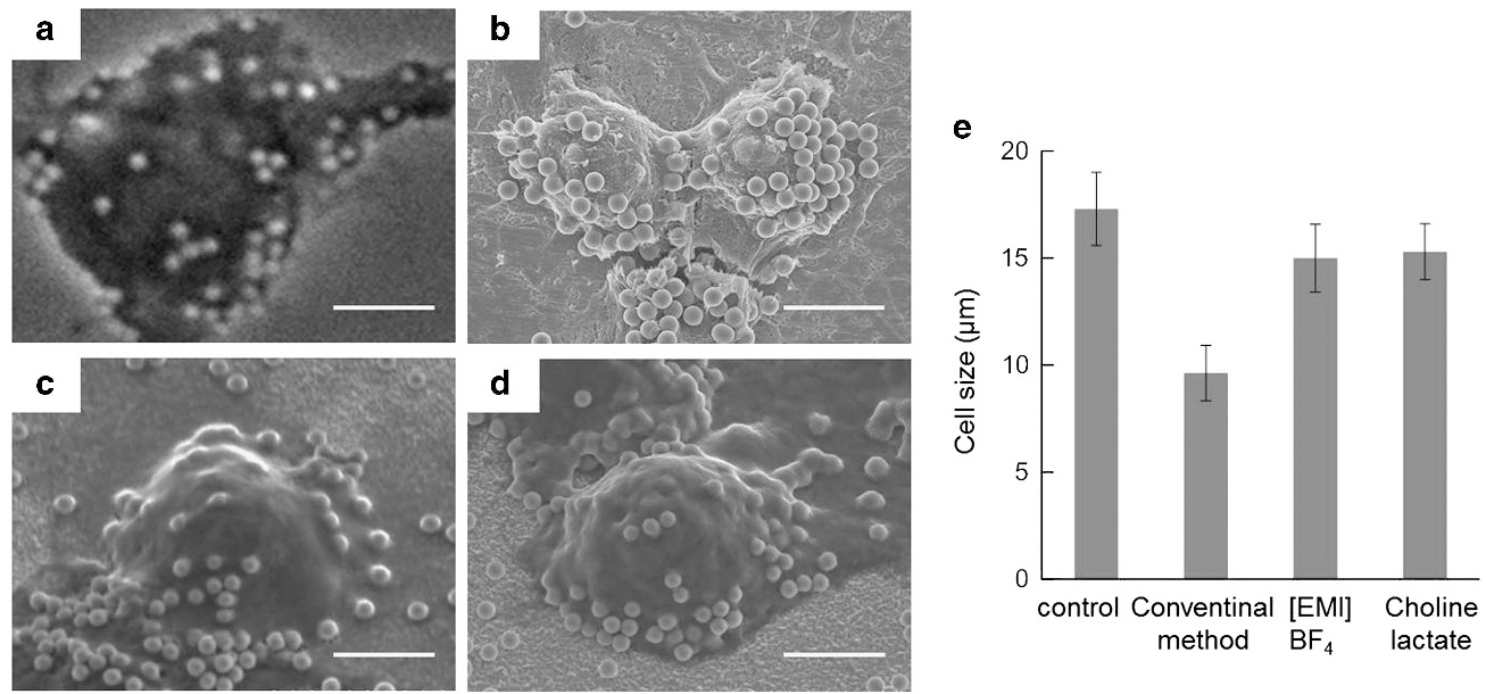

Figure 4 Cellular uptake of silica particles by RAW264 cells. The cells were incubated with silica particles for 60 min, and after being fixed, the cells were observed by (a) phase contrast microscopy, (b) SEM using conventional method, and SEM using (c) [EMI]BF 4 or (d) choline lactate. The scale bars represent $5 \mu \mathrm{m}$. (e) Sizes of the cells measured by each method $(n=10)$. A full color version of this figure is available at Polymer Journal online.

a

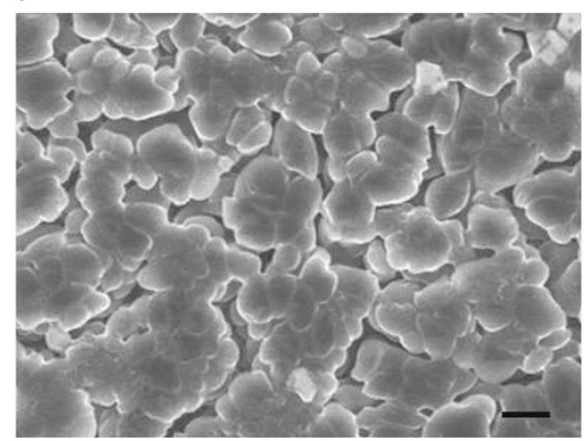

b

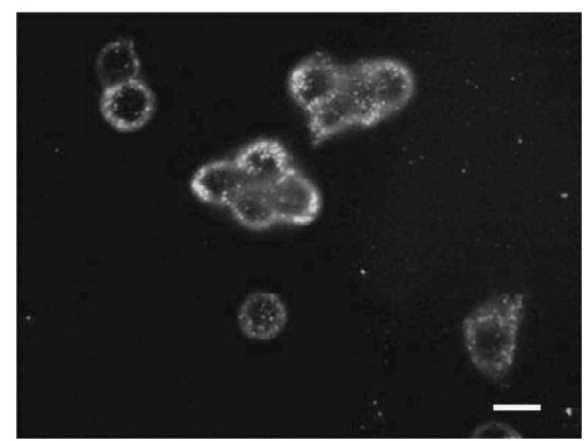

Figure 5 (a) Stability of the $\gamma$-PGA-Phe NPs in a $2 \mathrm{wt} \%$ choline lactate solution. The sample was dried under vacuum for $1 \mathrm{~h}$ followed by observation using SEM. Scale bar represents $1 \mu \mathrm{m}$. (b) Cellular uptake of Alexa488-NPs by RAW264 cells. The cells were incubated with Alexa488-NPs for $1 \mathrm{~h}$, fixed and then dipped into $1 \mathrm{wt} \%$ choline lactate for $15 \mathrm{~s}$ followed by drying under vacuum for $1 \mathrm{~h}$. The scale bar represents $10 \mu \mathrm{m}$. A full color version of this figure is available at Polymer Journal online. 

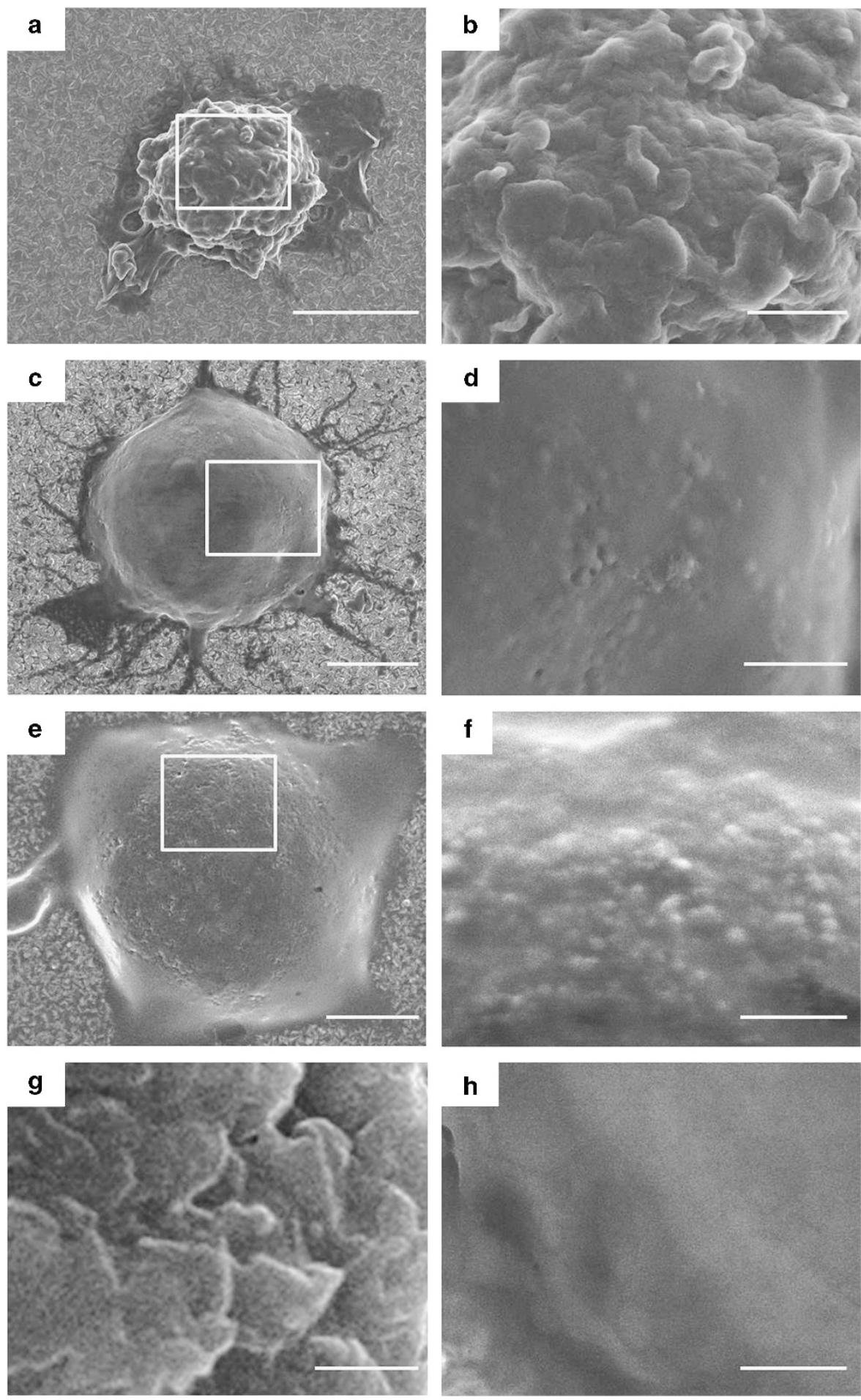

Figure 6 Observation of cellular uptake of the $\gamma$-PGA-Phe NPs by RAW264 cells. (a) The sample was pretreated using the conventional method followed by SEM observation. (c) The sample was dipped into $1 \mathrm{wt} \%$ choline lactate for $15 \mathrm{~s}$ followed by SEM observation. (e) The cells were incubated with QD-NPs, dipped into $1 \mathrm{wt} \%$ choline lactate for $15 \mathrm{~s}$, and observed by SEM. (b, $\mathbf{d}$ and $\mathbf{f}$ ) are the high magnification images of the square areas in a, $\mathbf{c}$ and $\mathbf{e}$, respectively. (g) The cells were treated using the conventional method followed by SEM observation. (h) The cells were treated with the ionic liquid followed by SEM observation. The scale bars in $\mathbf{a}, \mathbf{c}$ and e represent $5 \mu \mathrm{m}$. The scale bars in $\mathbf{b}, \mathbf{d}, \mathbf{f}, \mathbf{g}$ and $\mathbf{h}$ represent $1 \mu \mathrm{m}$.

in a hydrated state (Figure 1b) due to the replacement of water with the ionic liquid. As previously reported, the ionic liquid method can be used to observe the wet state of samples that exhibit quite different morphologies under the dry conditions compared with those under wet conditions. ${ }^{17,26}$ The water in the $\gamma$-PGA-Phe NPs might be replaced with the ionic liquid during pretreatment, which would result in the existence of a hydrated state. For detailed observations, the concentration of the ionic liquid is crucial. To observe NPs and 
a
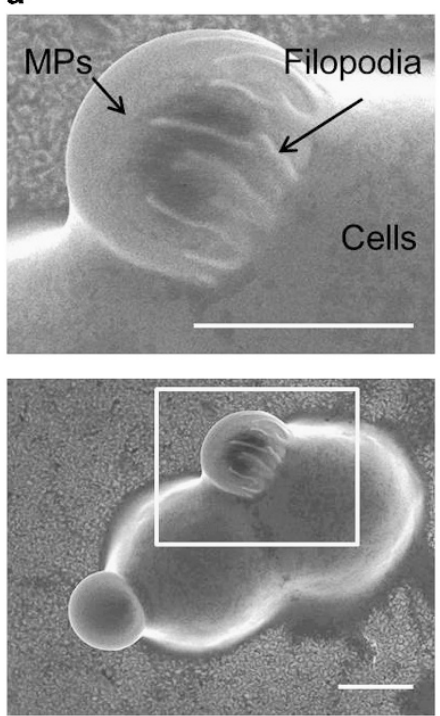

b
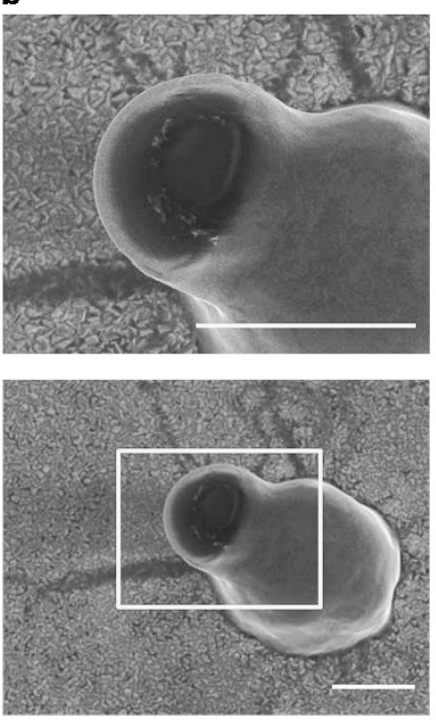

c
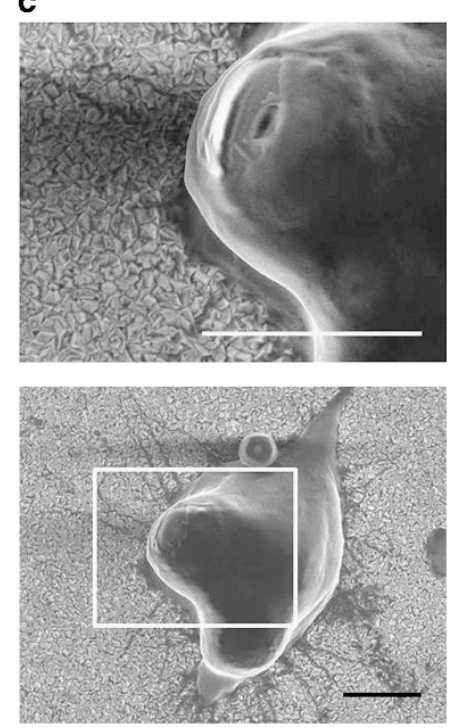

Figure 7 Representative images of cellular uptake of the $\gamma$-PGA-Phe MPs by RAW264 cells using their filopodia. The sample was immersed in a 1 wt\% choline lactate solution for $15 \mathrm{~s}$ and observed by SEM. The cells were incubated with MPs for (a) $10 \mathrm{~min}$, (b) $30 \mathrm{~min}$ and (c) $60 \mathrm{~min}$. The upper images are high magnifications of the square areas in the lower images. The scale bars represent $5 \mu \mathrm{m}$.

cellular uptake of NPs, the ionic liquid should be thinly covered the surface of samples. Therefore, we further optimized the concentration of choline lactate, and found that samples did not show charge-up effect when they were treated with $1 \mathrm{wt} \%$. We also observed the $\gamma$-PGA-Phe MPs using the ionic liquid method and confirmed that these MPs were also stable in the ionic liquid (data not shown).

The effect of the ionic liquid treatment on the observation of the cellular uptake of the $\gamma$-PGA-Phe NPs was evaluated using fluorescence-labeled $\gamma$-PGA-Phe NPs (Alexa488-NPs). The sample was dipped into $1 \mathrm{wt} \%$ choline lactate for $15 \mathrm{~s}$ and dried under vacuum for $1 \mathrm{~h}$ before observation by confocal fluorescence microscopy. The fluorescence images indicated that the ionic treatment did not affect the observation of the cellular uptake of Alexa488-NPs. No damage, such as aggregation or dissolution of the NPs, was observed (Figure 5b). Therefore, the cellular uptake of the $\gamma$-PGA-Phe NPs was observed by SEM using choline lactate in the ionic liquid method for the following experiments.

\section{Observation of the cellular uptake of NPs and MPs using the ionic liquid method}

To investigate the cellular uptake of vaccine carriers under wet conditions by SEM, we employed the ionic liquid method using choline lactate. As previously described, the pretreatment only required a few minutes, and the method was quite simple, ${ }^{18}$ which are advantageous compared with conventional methods including wet SEM and environmental SEM. The sample treated with the ionic liquid could be observed without coating with metal or carbon, which indicates that a very thin ionic liquid layer coated the sample surface ${ }^{17}$ resulting in the observation of the hydrated state of cells (Figure 6h). However, the sample had to be treated with metal, such as osmium, to avoid the charge-up effect during observation using the conventional method (Figure 6g).

First, the RAW264 cells were incubated with the $\gamma$-PGA-Phe NPs for $1 \mathrm{~h}$ followed by observation using SEM. As expected, the $\gamma$-PGA-Phe NPs were observed on the surface of the cells (Figures $6 \mathrm{c}$ and $\mathrm{d}$ ). However, the $\gamma$-PGA-Phe NPs were not observed when the sample was treated by the conventional method (Figures 6a and b) because the $\gamma$-PGA-Phe NPs collapsed during the dehydration process, and also damaged the sample (that is, shrinkage, change in the morphology). Therefore, this ionic liquid method exhibits high potential for observing polymeric particles whose structure is sensitive to the conventional pretreatment process. However, there is a limitation; the contrast of the $\gamma$-PGA-Phe NPs was insufficient for a detailed evaluation of the cellular uptake. When the particles contain atoms with a higher atomic number than carbon, the contrast was improved because a large number of secondary electrons were emitted from these atoms. ${ }^{18}$ Therefore, to observe the cellular uptake of the $\gamma$-PGA-Phe NPs in detail, the $\gamma$-PGA-Phe NPs should be labeled with heavy atoms, such as silicon or a metal.

The cellular uptake of the $\gamma$-PGA-Phe NPs by the RAW264 cells was clearly observed when the QD-NPs were pulsed to the cells (Figures 6e and f). By immobilizing the QDs, the contrast of the NPs was improved compared with the $\gamma$-PGA-Phe NPs without immobilization of the QD (Figures 6d and f). The QDs were mainly composed of heavy atoms, such as cadmium, selenium and zinc, and a large number of secondary electrons were emitted from these atoms, resulting in high contrast images.

Interestingly, the cellular uptake of the $\gamma$-PGA-Phe MPs was clearly determined using the ionic liquid method. The RAW264 cells were incubated with the $\gamma$-PGA-Phe MPs for $1 \mathrm{~h}$, fixed with formalin, and then dipped into $1 \mathrm{wt} \%$ choline lactate for $15 \mathrm{~s}$. As shown in Figure 7, the filopodia that covered the $\gamma$-PGA-Phe MPs to uptake them were observed. Therefore, we can evaluate the cellular uptake of vaccine carriers in detail by SEM using the ionic liquid method because no morphological changes were noted, and the hydrated state of the cells could be observed. The cells can be observed without staining using wet SEM. However, the contrast is insufficient, which is a limitation for the evaluation of the interactions between vaccine carriers and cells. ${ }^{10}$ Several studies have demonstrated that cellular uptake of polystyrene particles ${ }^{27}$ or micro-organisms ${ }^{28}$ with filopodia can be observed by SEM even though these studies were conducted under dry conditions and thus different from the native state. By contrast, 
our method allowed for the observation of the filopodia, which is $\sim 300 \mathrm{~nm}$ in width under hydrated conditions. Therefore, this method should be useful for gaining a mechanistic understanding of the interactions between vaccine carriers and cells.

The time dependence of the cellular uptake of the $\gamma$-PGA-Phe MPs was evaluated using the ionic liquid method. The RAW264 living cells were incubated with the $\gamma$-PGA-Phe MPs for 10, 30 and $60 \mathrm{~min}$, and after fixation, these cells were treated with the ionic liquid. Figure 7 shows representative images of the cellular uptake at each time point. First, the $\gamma$-PGA-Phe MPs were covered with filopodia, and the cell membrane gradually covered the $\gamma$-PGA-Phe MPs. To the best of our knowledge, this is the first time that cellular uptake of vaccine carriers was observed in a time-dependent manner with high contrast and without any damage. Recently, various ionic liquids have been developed, and therefore, we can select suitable ionic liquids for observing biomaterials, such as cells and tissues. ${ }^{17}$ This method also provides a strategy for observing vaccine carriers that are unstable under dry conditions. Because the interactions between biomaterials, such as cells, tissues and organic or inorganic materials, have attracted much attention for the development of novel pharmaceutical substances and therapeutic devices, our results suggest that this ionic liquid method can be used as a simple and high contrast evaluation method in this field of study.

\section{CONCLUSIONS}

We evaluated the cellular uptake of the $\gamma$-PGA-Phe NPs and $\gamma$-PGA-Phe MPs using an ionic liquid method. The contrast of the $\gamma$-PGA-Phe NPs was improved by immobilizing QDs. In addition, detailed observations of the cellular uptake of the $\gamma$-PGA-Phe MPs were achieved, which demonstrated that this technique can be applied to the medical and biological fields for evaluating biological samples at a high resolution. We believe that this approach will be useful in the life sciences.

\section{ACKNOWLEDGEMENTS}

This work was supported by Core Research for Evolutional Science and Technology (CREST) from the Japan Science and Technology Agency (JST) and partially supported by a Grant-in-Aid for Scientific Research (S) from the Ministry of Education, Culture, Sports, Science, and Technology (23225004).

1 Reddy, S. T., Swartz, M. A. \& Hubbell, J. A. Targeting dendritic cells with biomaterials: developing the next generation of vaccines. Trends Immunol. 27, 573-579 (2006).

2 Rice-Ficht, A. C., Arenas-Gamboa, A. M., Kahl-McDonagh, M. M. \& Ficht, T. A Polymeric particles in vaccine delivery. Curr. Opin. Microbiol. 13, 106-112 (2010).

3 Reddy, S. T., Vlies, A. J., Simeoni, E., Angeli, V., Randolph, G. J., O'Neil, C. P., Lee, L. K., Swartz, M. A. \& Hubbell, J. A. Exploiting lymphatic transport and complement activation in nanoparticle vaccines. Nat. Biotechnol. 25, 1159-1164 (2007).

4 Oyewumi, M. O., Kumar, A. \& Cui, Z. Nano-microparticles as immune adjuvants: correlating particle sizes and the resultant immune responses. Expert Rev. Vaccines $\mathbf{9}$, 1095-1107 (2010)
5 O'Hagen, D. T. \& Valiante, N. M. Recent advances in the discovery and delivery of vaccine adjuvants. Nat. Rev. Drug Discov. 2, 727-735 (2003).

6 Panyam, J., Zhou, W. Z., Prabha, S., Sahoo, S. K. \& Labhasetwar, V. Rapid endo-lysosomal escape of poly(DL-lactide-co-glycolide) nanoparticles: implications for drug and gene delivery. FASEB J 16, 1217-1226 (2002).

7 Dixon, A. E., Damaskinos, S. \& Atkinson, M. R. A scanning confocal microscope for transmission and reflection imaging. Nature 351, 551-553 (1991).

8 Diwan, M., Elamanchili, P., Lane, H., Gainer, A. \& Samuel, J. Biodegradable nanoparticle mediated antigen delivery to human cord blood derived dendritic cells for induction of primary T cell responses. J. Drug Target 11, 495-507 (2003).

9 Thiberge, S., Zik, O. \& Moses, E. An apparatus for imaging liquids, cells, and other wet samples in the scanning electron microscopy. Rev. Sci. Instrum. 75, 2280-2289 (2004)

10 Thiberge, S., Nechushtan, A., Sprinzak, D., Gileadi, O., Behar, V., Zik, O., Chowers, Y., Michaeli, S., Schlessinger, J. \& Moses, E. Scanning electron microscopy of cells and tissues under fully hydrated conditions. Proc. Natl Acad. Sci. USA 101, 3346-3351 (2004).

11 Bogner, A., Thollet, G., Basset, D., Jouneau, P. H. \& Gauthier, C. Wet STEM: a new development in environmental SEM for imaging nano-objects included in a liquid phase. Ultramicroscopy 4, 290-301 (2005).

12 Wilkes, J. S. \& Zaworotko, M. J. Air and water stable 1-ethyl-3-methylimidazolium based ionic liquids. J. Chem. Soc., Chem. Commun. 13, 965-967 (1992).

13 Welton, T. Room-temperature ionic liquids. Solvents for synthesis and catalysis. Chem. Rev. 99, 2071-2084 (1999).

14 Hagiwara, R. \& Ito, Y. Room temperature ionic liquids of alkylimidazolium cations and fluoroanions. J. Fluorine Chem. 105, 221-227 (2000).

15 Kuwabata, S., Kongkanand, A., Oyamatsu, D. \& Torimoto, T. Observation of ionic liquid by scanning electron microscope. Chem. Lett. 35, 600-601 (2006).

16 Ishigaki, Y., Nakamura, Y., Takehara, T., Nemoto, N., Kurihara, T., Koga, H. Nakagawa, H., Takegami, T., Tomosugi, N., Miyazawa, S. \& Kuwabata, S. Ionic liquid enables simple and rapid sample preparation of human culturing cells for scanning electron microscope analysis. Microsc. Res. Tech. 74, 415-420 (2011)

17 Tsuda, T., Nemoto, N., Kawakami, K., Mochizuki, E., Kishida, S., Tajiri, T. Kushibiki, T. \& Kuwabata, S. SEM observation of wet biological specimens pretreated with room-temperature ionic liquid. ChemBioChem. 12, 2547-2550 (2011).

18 Shima, F., Kawakami, K., Akagi, T., Mochizuki, E., Tsuda, T., Kuwabata, S. \& Akashi, M. Simple observation of the interaction between nanoparticles and cells by scanning electron microscopy employing ionic liquid. Bull. Chem. Soc. Jpn 86, 153-158 (2013)

19 Matsusaki, M., Hiwatari, K., Higashi, M., Kaneko, T. \& Akashi, M. Stably-dispersed and surface-functional bionanoparticles prepared by self-assembling amphipathic polymers of hydrophilic poly $(\gamma$-glutamic acid) bearing hydrophobic amino acids. Chem. Lett. 33, 398-399 (2004)

20 Uto, T., Akagi, T., Hamasaki, T., Akashi, M. \& Baba, M. Modulation of innate and adaptive immunity by biodegradable nanoparticles. Immunol. Lett. 125, 46-52 (2009).

21 Akagi, T., Piyapakorn, P. \& Akashi, M. Formation of unimer nanoparticles by controlling the self-association of hydrophobically modified poly(amino acid)s. Langmuir $\mathbf{2 8}$ 5249-5256 (2012).

$22 \mathrm{Kim}$, H., Akagi, T. \& Akashi, M. Preparation of size tunable amphiphilic poly(amino acid) nanoparticles. Macromol. Biosci. 9, 842-848 (2009).

23 Shima, F., Akagi, T., Uto, T. \& Akashi, M. Manipulating the antigen-specific immune response by the hydrophobicity of amphiphilic poly $(\gamma$-glutamic acid) nanoparticles. Biomaterials 34, 9709-9716 (2014).

24 Shima, F., Akagi, T. \& Akashi, M. The role of hydrophobicity in the disruption of erythrocyte membrane by nanoparticles composed of hydrophobically modified poly( $\gamma$-glutamic acid). J. Biomater. Sci. Polym. Ed. 25, 203-210 (2014).

25 Shima, F., Uto, T., Akagi, T., Baba, M. \& Akashi, M. Size effect of amphiphilic poly $(\gamma$-glutamic acid) nanoparticles on cellular uptake and maturation of dendritic cells in vivo. Acta Biomater. 9, 8894-8901 (2013).

26 Arimoto, S., Sugimura, M., Kageyama, H., Torimoto, T. \& Kuwabata, S. Development of new techniques for scanning electron microscope observation using ionic liquid. Electrochim. Acta 53, 6228-6234 (2008).

27 Champion, J. A. \& Mitragotri, S. Role of target geometry in phagocytosis. Proc. Nat/ Acad. Sci. USA 13, 4930-4934 (2006).

28 Walters, M. N-I., Papadimitriou, J. M. \& Robertson, T. A. The surface morphology of the phagocytosis of micro-organisms by peritoneal macrophages. J. Pathol. 118, 221-226 (1976) 